\title{
Digital holographic microscopy as a screening technology for diabetes
}

\section{Ana Doblas, Jorge Garcia-Sucerquia, Genaro Saavedra, Manuel Martinez-Corral}

Ana Doblas, Jorge Garcia-Sucerquia, Genaro Saavedra, Manuel MartinezCorral, "Digital holographic microscopy as a screening technology for diabetes," Proc. SPIE 10997, Three-Dimensional Imaging, Visualization, and Display 2019, 109970K (14 May 2019); doi: 10.1117/12.2523416

SPIE Event: SPIE Defense + Commercial Sensing, 2019, Baltimore, Maryland, United States 


\title{
Digital holographic microscopy as a screening technology for diabetes \\ Ana Doblas ${ }^{\mathrm{a}, *}$, Jorge Garcia-Sucerquiab, Genaro Saavedrac, Manuel Martinez-Corral ${ }^{\mathrm{c}}$ \\ ${ }^{a}$ Department of Electrical and Computer Engineering, University of Memphis, Memphis, TN, USA

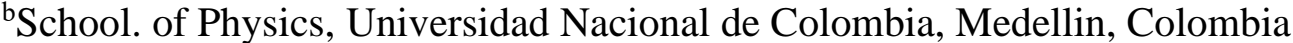 \\ ${ }^{c}$ Department of Optics, University of Valencia, Burjassot, Spain
}

\begin{abstract}
Label-free quantitative phase imaging (QPI) is the hallmark of digital holographic microscopy (DHM). One of the most interesting medical applications of QPI-DHM is that it can be used to analyze illnesses in which the refractive index or/and the morphology of cells/tissues are distorted, from the acquisition of a single image. In this contribution, we obtain the phase maps of red blood cells (RBCs) samples of patients suffering from diabetes mellitus type 1 (DM1) by using a DHM. Our experimental results show that the measured phase values are significantly different between control non-diabetic and diabetic patients. The high correlation coefficient between the phase and the glycated hemoglobin (HbA1C) values determined by the gold standard method to screen diabetes and the clear separation between the two groups indicate that DHM may potentially be used to evaluate long-term glycemic control in diabetic patients as well as to diagnose diabetes.
\end{abstract}

Keywords: digital holographic microscopy, phase retrieval, red blood cells, diabetes.

\section{INTRODUCTION}

Diabetes mellitus (DM) is one of the top ten leading cause of death over the world. DM is a disease that includes a series of metabolic alterations that affect different organs and tissues due to a deficient production of insulin by the endocrine pancreas or by abnormal insulin action in peripheral tissues ${ }^{1,2}$. As a general classification, DM is classified into four broad categories $^{3,4}$ : type 1 , type 2, gestational diabetes and others. The first types (type 1 and type 2 ) are the most prevalent and responsible for most of the health care cost attributed to this disease. Type 1 diabetes mellitus (T1DM) is characterized by the total loss of insulin ${ }^{5}$ and, consequently, T1DM patients require lifelong insulin injections since there are no alternative hormones in the organism mimicking the effect of insulin. Patient with type 2 DM (T2DM) are in a first phase of the disease insulin-resistant and the pancreas compensates by secreting high levels of the hormone. However, over time, T2DM often develops into T1DM. One characteristic of T1DM is that patients with T1DM present prominent fluctuations in blood glucose, with longer periods of hyperglycemia compared to non-diabetic individuals ${ }^{6,7}$. This results over time in the development of secondary complications that affect mainly the retina, kidneys, nerves and cardiovascular system ${ }^{8}$. The gold standard parameter to evaluate periodically glycemic control in patients with diabetes is the determination of glycated hemoglobin (HbA1C) $)^{9,10}$. The concentration of HbA1C indicates the level of exposure to persistent hyperglycemia over the last 2-3 months, coinciding with the half-life of hemoglobin (approximately 120 days), and it is measured by highperformance liquid chromatography (HPLC).

This study is devoted to analyze the alterations of the phase-map distributions using digital holographic microscopy (DHM) in erythrocytes of patients with diabetes compared to non-diabetic controls ${ }^{11}$. In $\mathrm{DHM}^{12-15}$, the reconstruction of the complex wavefield (amplitude and phase) of a microscopic sample is achieved by applying two processes in cascade. Firstly, one records the interference pattern between the complex amplitude distribution of the image of an object field produced by a host microscope and a reference plane wave. This stage is commonly named as the optical recording of a digital hologram. The second stage is the numerical (e.g. computational) recovery of the wavefield scattered by the object. After applying a numerical reconstruction approach, this technique allows us both numerical refocusing to different planes and the quantitative map of the phase distribution on the specimen under research. Thanks to these two features, DHM has become a crucial method for investigating biological cells ${ }^{16-21}$. The motivation of this work is based in two previous studies $^{22,23}$. In the first, Mazarevica et al..$^{22}$ reported that the refractive properties of the red blood cells (RBCs) in DM patients greatly differs from those of the healthy subjects. In fact, they demonstrated a relationship between the refractive index and $\mathrm{pH}$ level for diabetic and healthy donors. The second shows that variations in plasma glucose cause refractive-

*adoblas@memphis.edu;

Three-Dimensional Imaging, Visualization, and Display 2019, edited by Bahram Javidi

Jung-Young Son, Osamu Matoba, Proc. of SPIE Vol. 10997, 109970K · C 2019

SPIE · CCC code: $0277-786 X / 19 / \$ 18 \cdot$ doi: $10.1117 / 12.2523416$

Proc. of SPIE Vol. 10997 109970K-1 
index changes in eyes of DM subjects and, as a result, transient myopia or hyperopia is derived ${ }^{23}$. As a proof-of-concept study, we have found that found that phase distribution of the RBC has the potential to be used to quantify persistent hyperglycemia states and, therefore even to be adopted as a screening method to diagnose abnormal hyperglycemia since there is a strong correlation between the measured phase and the HbA1C values. Note that in 1952, Barer ${ }^{24}$ demonstrated that hemoglobin concentration is linearly dependent on phase measurement.

\section{DIGITAL HOLOGRAPHIC MICROSCOPY (DHM)}

As stated above, DHM can be understood as two independent stages: the optical recording of the hologram and, consequently, its reconstruction to retrieve both the amplitude and phase distributions of the sample under research. In this section, we provide a thorough description of the optical stage in DHM systems. In the recording stage, only the effects of diffraction and interference are taken into account. One of the most common optical configuration used in DHM is the modified Mach-Zenhder interferometer, shown in Fig. 1(a). The light proceeding from a laser of wavelength $\lambda$ is collimated by a converging lens (CL) and the resultant plane beam impinges a cubic beam splitter (BS1). The split beams are usually known as the object and the reference waves. From Fig. 1 it is easy to realize that a conventional optical microscope, commonly called as the host microscope, is engaged in the object arm of the interferometer. The wavefield scattered by the specimen of amplitude distribution $\boldsymbol{O}(\boldsymbol{x})$ is imaged by an imaging system composed by an infinity-corrected microscope objective (MO) and a tube lens (TL). Since the object is set at the front-focal plane (FFP) of the MO, the image of the sample, whose complex amplitude distribution is $U_{I P}(\boldsymbol{x})$, is then obtained at the back-focal-plane (BFP) of the TL. Note that this plane is the image plane (IP) of the host microscope.

Then, the irradiance pattern recorded by the sensor, called hologram, is the result of the optical interference between a tilted plane wave with the $U_{I P}(\boldsymbol{x})$ wavefield propagated by a distance $z$ from the IP. Without lack of generality, we have considered that the sensor plane coincides with the IP in Fig. 1a. This consideration does not affect the achieved lateral resolution of the DHM system ${ }^{25}$ and simplify the reconstruction steps. Further, to retrieve the sample information using a single-shot approach, the DHM systems operates in an off-axis configuration. This means that both the beam-splitter BS2 and the mirror M2, which both reflect the reference wave $R$, are slightly tilted such as the reference wavefield interferes with the object wave $O$ with an angle of $\boldsymbol{\theta}=\left(\theta_{x}, \theta_{x}\right)$ with respect the optical axis, $R(\boldsymbol{x} ; \boldsymbol{\theta})$. Thereby, the hologram $H(\boldsymbol{x})$ at the IP plane is

$$
H(\boldsymbol{x})=\left|U_{I P}(\boldsymbol{x})\right|^{2}+|R(\boldsymbol{x} ; \boldsymbol{\theta})|^{2}+U_{I P}(\boldsymbol{x}) R^{*}(\boldsymbol{x} ; \boldsymbol{\theta})+U_{I P}^{*}(\boldsymbol{x}) R(\boldsymbol{x} ; \boldsymbol{\theta}),
$$

where $\boldsymbol{x}=(x, y)$ are the spatial transverse coordinates, $|\cdot|^{2}$ represents the square modules and the sign * is the complex conjugate operation. From Eq. (1), one realizes that the hologram is composed by four terms. The first two terms do not carry any information about the phase of the object and the angle of the reference wave and they produce, when Fourier transformed, the zero-order of diffraction (e.g. the DC term). The DC term is always placed at the center of the Fourier transform of the hologram. The third and fourth terms are identified as the \pm 1 diffraction orders in the Fourier domain, respectively, and encode the whole sample information, both in amplitude and phase. Thanks to the off-axis configuration, the \pm 1 diffraction orders are arranged symmetrically around the DC term in the Fourier space. According to wellestablished reconstruction methods, the object information can be obtained by spatially filtering out the $+1 \mathrm{term}^{26}$.

Now, let us focus our attention to the object arm and the complex amplitude distribution of the object at the IP, $U_{I P}(x)$. Under the paraxial regime, the $U_{I P}(\boldsymbol{x})$ distribution can be computed by application in cascade of ABCD transformation ${ }^{27}$. After some mathematical manipulations, the $U_{I P}(\boldsymbol{x})$ distribution is given by

$$
U_{I P}(x)=\frac{1}{M^{2}} e^{\mathrm{i} k\left(2 f_{M O}+d+f_{T L}\right)} \exp \left(\mathrm{i} \frac{k}{2 C}|x|^{2}\right) \times\left\{O\left(\frac{x}{M}\right) \otimes_{2} \tilde{p}\left(\frac{x}{\lambda f_{T L}}\right)\right\},
$$

where $\boldsymbol{x}=(x, y)$ are the spatial transverse coordinates, $k=2 \pi / \lambda$ is the illumination wave number, $\otimes_{2}$ denotes the 2D convolution operator, $M=-f_{T L} / f_{M O}$ stands for the lateral magnification of the host microscope, which only depends on the focal lengths of the $\mathrm{MO}\left(f_{M O}\right)$ and the TL $\left(f_{T L}\right)$, and $\tilde{p}(\bullet)$ is the 2D Fourier Transform of the amplitude transmittance of the pupil. In Eq. (2), the quadratic phase term of curvature radius $C=\left(f_{T L}^{2}\right) /\left(f_{T L}-d\right)$ is well-known recognized in DHM and associated with the use of a non-telecentric imaging system ${ }^{28-30}$. This phase factor appears when the host microscope in the DHM follows a non-telecentric geometry, $d \neq f_{\text {IL }}$ being $d$ the distance between the BFP of the MO and the TL. As we have shown recently ${ }^{29,30}$, the existence of this quadratic phase term presents significant drawbacks on 
phase measurements and the resolution capability introduced by a non-telecentric geometry of the imaging system. This additional phase factor turns the DHM into a shift-variant system which distorts phase measurements ${ }^{28,29}$. Besides the above, the non-telecentric DHM also presents limitations in amplitude contrast imaging. In fact, the presence of the quadratic phase term increases the size of the \pm 1 terms in the Fourier spectrum of the hologram. This broadening makes harder the spatial filtering of the +1 term in the reconstruction stage and can provide a deteriorated reconstructed image that the DHM system becomes a shift-variant imaging system ${ }^{30}$.

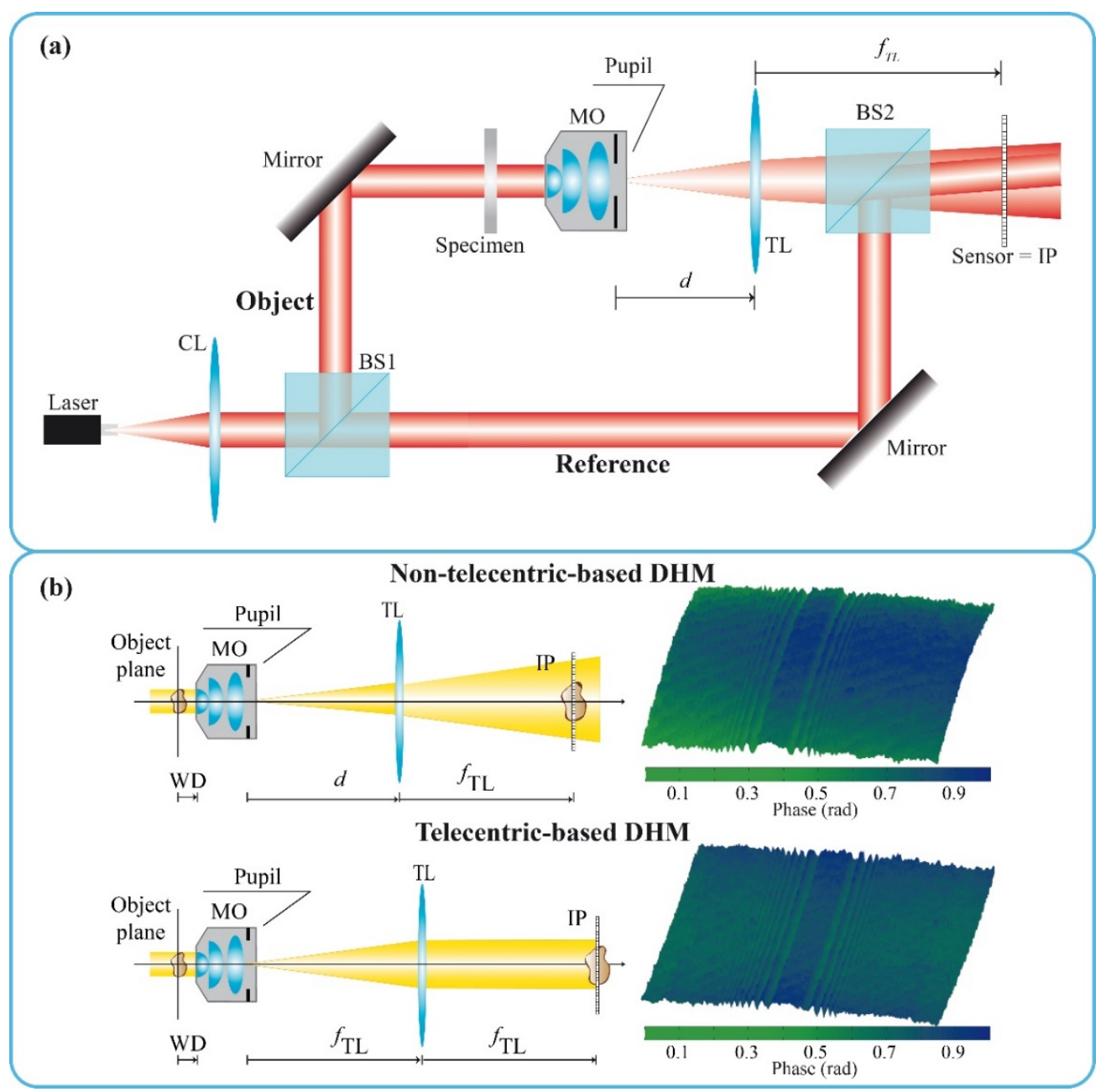

Fig. 1 (a) Scheme of an off-axis transmission DHM. In a general case, the MO and the TL are arranged in non-telecentric mode $\left(d \neq f_{\mathrm{TL}}\right)$. (b) Illustration of the presence of a spherical distortion due to a non-telecentric geometry of the object arm in the 3D-pseudocolor QPI of a phase sample composed by radial stripes of different width. In the first row the DHM optical configuration does not operate in the telecentric regime $\left(C=8000 \mathrm{~mm}\right.$ and $\left.d=f_{\mathrm{TL}}-5 \mathrm{~mm}=195 \mathrm{~mm}\right)$ and the spherical factor introduced by the non-telecentric configuration has been eliminated via a posteriori numerical approach. In the second row the optical configuration operates at telecentric regime $\left(C=\infty\right.$ and $\left.d=f_{\mathrm{TL}}\right)$. Note that in this case there is no presence of the remaining quadratic phase factor. The image area in the 3D QPIs is $0.521 \times 1.203 \mathrm{~mm}^{2}$.

Because the majority of off-axis DHMs reported in the literature operate in non-telecentric regime, we revise the effect of using a non-telecentric geometry in QPI-DHM. As shown in Fig, 1(b), the value of the radius of curvature $C$ of the quadratic phase factor $\exp \left(\mathrm{i} k|x|^{2} / 2 C\right)$ in Eq. (2) depends on the distance between the MO aperture stop and the TL plane, $d$. Notwithstanding, this $C$ parameter can be tuned by changing the separation between the $\mathrm{MO}$ and the TL. The direct consequence of this quadratic phase term is that the imaging system becomes shift-variant ${ }^{28,29}$. This means that the accuracy of the QPI measurements strongly depends on the position of the sample in the field of view (FOV). In non-telecentric QPI-DHM, this undesired quadratic phase factor is commonly eliminated a-posteriori via computational approaches. Mainly, the numerical approaches used to remove the phase factor require the accurate computation of its center and radius of curvature to cancel out its effect on QPI measurements. However, even a minimum 
error in these parameters of the quadratic phase factor, perturbs the accuracy of the QPI-DHM, as shown in the second column of Fig. 1(b). To evaluate the shift-invariance property between non-telecentric- and telecentric- DHM systems, we have reconstructed the phase map of a phase sample constituted by stripes of different width and equal height made on a glass substrate. We recorded two different holograms: one when the DHM operates in a non-telencetric regime with a $C=8000 \mathrm{~mm}\left(d=f_{\mathrm{TL}}-5 \mathrm{~mm}=195 \mathrm{~mm}\right)$ and the second one when the DHM system performs at the telectric mode $\left(C=\infty\right.$ and $\left.d=f_{\mathrm{TL}}\right)$. The spherical phase factor introduced by the nontelecentric arrangement has been eliminated using the common a posteriori numerical approach ${ }^{31-33}$. Note that although the spherical phase factor has been removed, the retrieved phase map still shows the presence of a remaining phase curvature which leads to a noninvariant phase measurement, see first row in Fig. 1(b). Nonetheless, from the telecentric result (second row in Fig. 1b), one realizes that the phase measurement of the sample is homogeneous over the complete FOV since no presence of the remaining phase factor is observed. Note that this phase map is directly retrieved without applying any additional numerical process. Consequently, to ensure accurate QPI-DHM measurements (e.g. invariance of the phase measurement over the whole FOV) using a single-shot approach, which are without any doubt the desired feature in biological studies, the DHM must operate in telecentric mode.

\section{SCREENING DIABETES WITH A TELECENTRIC-BASED DHM}

Our goal is to analyze if there are alterations in phase maps of RBCs between DM patients and non-diabetic controls. Based on these preliminary studies ${ }^{22,23}$, we analyze the alterations of the distributions of the phase maps using telecentricDHM. To face this task, samples are obtained from both non-diabetic individuals (14 controls) and 29 patients with T1DM who are under treatment with insulin injections. In this study, we need two different kind of samples. To perform the clinical measurements of HbA1C using HPLC, blood extracted from the antecubital vein is required. The other sample is directly a capillary blood drop to smear it on a glass slide. These smeared blood slides as used as the specimen in our telecentric-based DHM of Fig. 1 to image RBCs from both the healthy-control group and the T1DM patients using a telecentric-DHM. Fig. 2(a) illustrates the corresponding phase maps. For better visualization of the differences between these maps, all images have been pseudo-colored using the same color scale. According to the color bar, it is easy to note that the phase values of healthy RBCs (first row of Fig. 2a) RBCs are significantly smaller than those obtained for the T1DM group (second row of Fig. 2b). Owing to the sample is prepared as a smearing of a blood drop, the measured phase includes contributions of both the RBCs and the plasma components. To remove the latter, the average value of the phase over the areas free of RBCs is subtracted from the total phase. This procedure has been applied to the phase maps show in Fig. 2a. Then, the phase value over the RBCs is essentially provided by the optical path length along the cells. To compare these measurements with the one provided by the gold standard method, it is necessary to calculate a mean phase value for each participant. For each cell we obtain the phase as the average value over an area of approximately $1.12 \times 1.12 \mu \mathrm{m}^{2}$ surrounding the centroid of the RBC. The mean phase value for each person is obtained as the average of the phase measurements of 20 individual erythrocytes. Fig. 2(b) shows the histograms of the RBC mean phase from healthy donors and diabetic patients whose average values were $3.15 \pm 0.13 \mathrm{rad}$ and $3.72 \pm 0.15 \mathrm{rad}$, respectively. It is clear that phase values in the T1DM group are significantly higher than the healthy control group. Applying the Mann-Whitney test ${ }^{34,35}$ to assess if there are differences between control and diabetes groups with the phase measurement, we find that using DHM it is possible to divide the population into groups of healthy or T1DM patients with a very high significance level ( $\mathrm{p}<$ 0.001). In addition, from the results depicted in Fig. $2 \mathrm{~b}$, it is clear that the phase measurements of each group do not overlap. In fact, the average phase values in healthy RBCs ranges 2.94 - $3.30 \mathrm{rad}$, while T1DM RBCs are 3.51 - 4.01 rad. Therefore, we can conclude that phase values above $3.40 \mathrm{rad}$ are an indication of diabetes. Although in this study, the whole analysis has been provided by using the phase measurement. It is worth to mention that the measured differences of the RBC phase values can be due to RI changes or morphological variations. To decouple both variations from the phase measurement, without any additional acquisition, we can measure the diameters of the RBCs for both groups of patients. Since the sample has been prepared leaving the RBCs free of mechanical constrains, one can assume that if a RBC experiences a morphological alteration, this modification will be present evenly along the three-directions. This means that if one cannot detect any change in the RBCs' diameter it could be claimed that the cell height of a RBC does not change as well and there is no morphological alteration. We measure and average the diameter size over 30 individual erythrocytes of healthy and T1DM RBCs; the average values are $7.3 \pm 0.6 \mu \mathrm{m}$ and $7.6 \pm 0.7 \mu \mathrm{m}$, respectively. Based on these measurements, one can conclude that no difference in the size can be detected for healthy and T1DM RBCs. Thus, the phase differences measured for both groups of RBCs, are mainly related to variations of the RI of the cells, as previously reported ${ }^{24}$. 

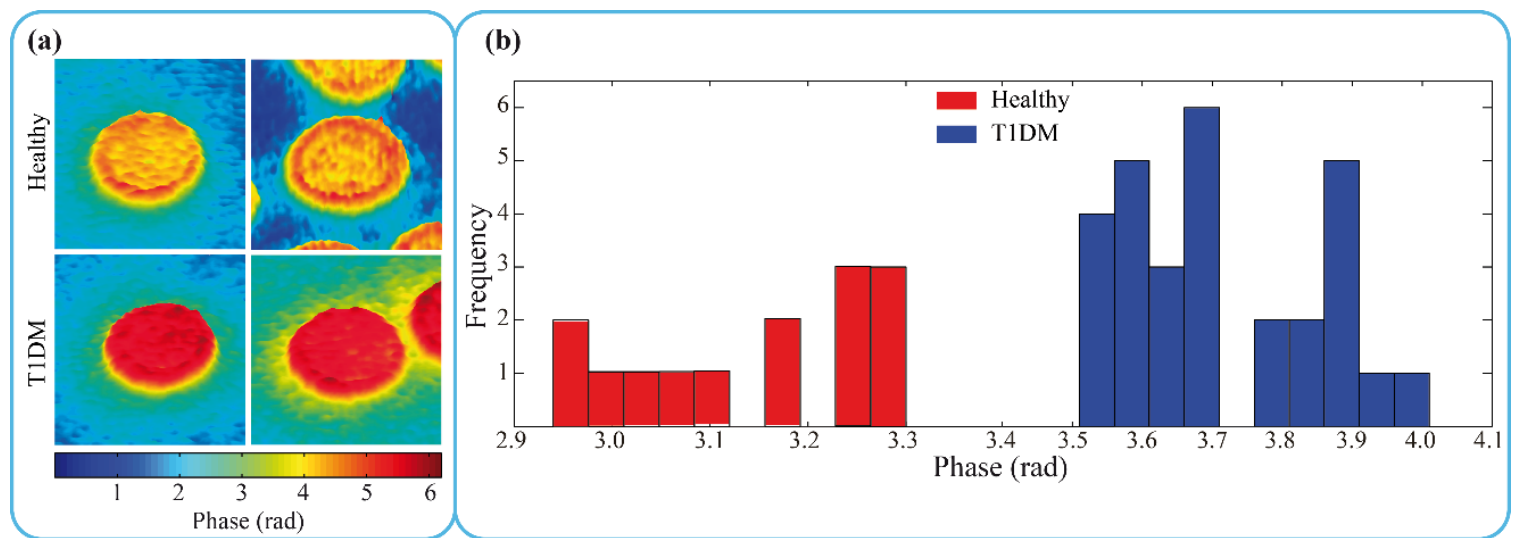

Fig. 2 (a) Pseudo-colored image of the phase maps of healthy RBCs (first row) and T1DM-RBCs (second row) measured by our telecentric-DHM. (b) Histograms of mean phase values of the healthy control group and the T1DM participants obtained in panel (a). In all cases in panel (a), the image area is $9.38 \times 9.38 \mu \mathrm{m}^{2}$.

In this study, we have also investigated if the measured phase values are correlated with HbA1C values. To this end, the phase values provided by our DHM are compared to the HbA1C data obtained by HPLC. The results can be observed in Fig. 3(a), where phase alterations are related to HbA1C changes. The rho correlation coefficients of Fig. 3(a) is 0.739 with $\mathrm{p}<0.01$. Since the correlation coefficient between $\mathrm{HbA1C}$ and phase values is high and there is a clear separation between these two groups in Fig. 3(a), one can claim that both HbA1C and phase may potentially be used to diagnose diabetes states as well as to evaluate long-term glycemic control in patients with diabetes. Finally, we compare both methods (DHM and HPLC) by showing the boxplots of the standardized phase and HbA1C values for healthy and T1DM RBCs, see Fig. 3(b). From these results, we observe that their standardized phase and HbA1C parameters in patients with diabetes are higher than the ones obtained for the healthy participants. The corresponding variation interquartile range $\left(\mathrm{IQR}=\mathrm{Q}_{3}-\mathrm{Q}_{1}\right)$ is calculated to measure the statistical dispersion of each indicator. Whereas the IQR coefficient of the phase measurement is of 1.89 for healthy subjects and 1.72 for diabetic patients, in the case of the standardized HbA1C the value is lower than the phase values (1.01 and 1.26 for healthy and diabetic group, respectively). This observation indicates that the gold standard technique presents less dispersion and, consequently, is less sensitive to errors. Notwithstanding, the range of the measurements for $\mathrm{HbA1C}$ is higher than the one for phase values, see Fig. 3(b). In particular, the phase range is between 2.78 and 3.38 in comparison to the HbA1C range, which is between 4.04 and 5.03 for healthy and diabetic groups, in that order. According to this, the HPLC technique provides a wider range for controlling diabetes. Nonetheless, we believe that DHM technique could be used as the first method since only a capillary blood drop is needed, it can be performed in any time and the phase measurement is obtained in a fairly instantaneous way.
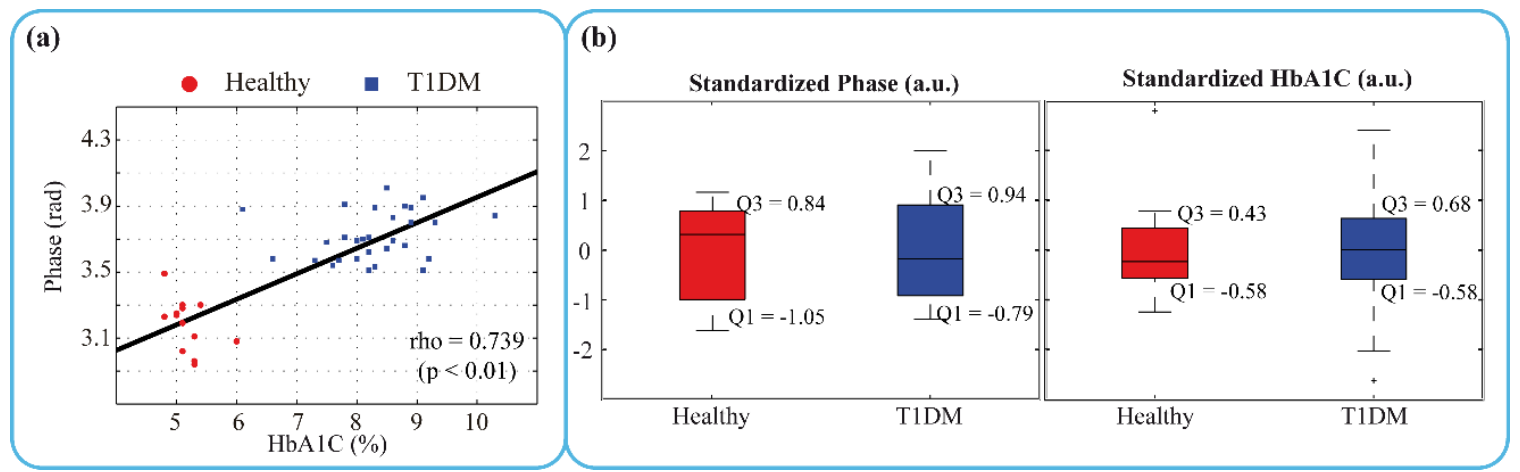

Fig. 3 Comparison between the phase and HbA1C values. (a) Phase vs HbA1 values and (b) Boxplot between the standardized phase and HbA1c (the gold standard) values. For better visualization of the tendency between the values in panel (a), the leastsquared best-fit line has been plotted and the rho correlation coefficient has been provided. 


\section{CONCLUSIONS}

In conclusion, we have demonstrated the use of telecentric-based DHM for DM screening. The utility of the DHM results from the possibility of providing quantitative phase maps as indicators of long-term blood sugar control similar to HbA1C. Although a strictly-medical research would require the analysis of much more test subjects, our results should be viewed as a proof-of-concept study. In addition, the proposed method shows great potential as optional tool to screen for diabetes because: (1) it is a minimally invasive technique since only a small drop of capillary blood is needed; (2) it can be performed at any time and the results can be obtained almost in real time; and (3) it is a widefield technique which can be easily implemented in a conventional microscope ${ }^{36}$, and (4) can be used to analyze illnesses in which the refractive index or/and the morphology are distorted. On the other hand, although HbA1C has been used clinically since the 1980s as a test of glycemic control in diabetes patients, the measurement of HbA1C provided by the HPLC method interferes with hemoglobinopathies and carbamylated $\mathrm{Hb}^{37}$. Apart from these drawbacks of the HbA1C measurement, the HPLC technique is also highly expensive.

\section{ACKNOWLEDGMENTS}

Authors acknowledge J. M. Adsuar Laboratory for providing the measurements of controls and T1DM patients with Type 1 diabetes followed at the Diabetes Reference Unit of Clinic University Hospital of Valencia, Spain. This work was supported by the University of Memphis, the Ministerio de Economia y Competitividad, Spain (Grant DPI2015-66458C2-1-R), the Generalitat Valenciana (Grant PROMETEOII/2014/072) and Universidad Nacional de Colombia (Hermes grant 19384).

\section{REFERENCES}

[1] Maahs, D.M., West, N.A., Lawrence, J.M., and Mayer-Davis, E.J., “Epidemiology of type 1 diabetes,” Endocrinol. Metab. Clin. North Am. 39, 481-497 (2010).

[2] Chen, L., Magliano, D.J., and Zimment, P.Z., "The worldwide epidemiology of type 2 diabetes mellitus-present and future perspectives,” Nat. Rev. Endocrinol. 8, 228-236 (2011).

[3] American-Diabetes-Association, "Diagnosis and classification of diabetes mellitus,” Diabetes Care 33(Suppl 1), S62-S69 (2010).

[4] Maraschin, J. de F., "Classification of diabetes.,” Adv. Exp. Med. Biol. 771, 12-91 (2012).

[5] Boitard, C., "Pancreatic islet autoimmunity,” Presse. Med. 41, e636-e650 (2012).

[6] Lee, Y.A., "Diabetes care for emerging adults: transition from pediatric to adult diabetes care systems," Metab., Ann. Pediatr. Endocrinol. 18, 106-110 (2013).

[7] Korytkowski, M.T., "In-patient management of diabetes: controversies and guidelines,” Indian J. Endocrinol. Metab. 17, S630-5 (2013).

[8] Forbes, J.M., and Cooper, M.E., “Mechanisms of diabetic complications,” Physiol. Rev. 93, 137-188 (2013).

[9] Consensus-Committed, "Consensus statement on the worldwide standardization of the hemoglobin A1C measurement,” Diabetes Care 30, 2399-2400 (2007).

[10] The-International-Expert-Committee, "International Expert Committee report on the role of the A1C assay in the diagnosis of diabetes,” Diabetes Care 32, 1327-1334 (2009).

[11] Doblas, A., Roche, E., Ampudia-Blasco, F.J., Martínez-Corral, M., Saavedra, G., and Garcia-Sucerquia, J., “Diabetes screening by telecentric digital holographic microscopy,” J. Microsc. 261, 285-290 (2016).

[12] Kreis, T., [Handbook of Holographic Interferometry] , Wiley (2004).

[13] Kim, M.K., "Principles and techniques of digital holographic microscopy," SPIE Rev. 1, 18005 (2010).

[14] Popescu, G., [Quantitative phase imaging of cells and tissues], McGraw-Hill (2011).

[15] Picart, P., and Li, J.-C., [Digital Holography], Wiley (2012).

[16] Kemper, B., Bauwens, A., Vollmer, A., Ketelhut, S., Langehanenberg, P., Müthing, J., Karch, H., and von Bally, G., "Label-free quantitative cell division monitoring of endothelial cells by digital holographic microscopy,” J. Biomed. Optics 15, 36009-36015 (2010).

[17] Puthia, M., Storm, P., Nadeem, A., Hsiung, S., and Svanborg, C., "Prevention and treatment of colon cancer by peroral administration of HAMLET (human $\alpha$-lactalbumin made lethal to tumour cells)," Gut 63, 132-142 
(2014).

[18] Anand, A., Chhaniwal, V.K., Patel, N.R., and Javidi, B., "Automatic identification of malaria-infected RBC with digital holographic microscopy using correlation algorithms,” IEEE Photonics Journal 4, 1456-1464 (2012).

[19] Wu, N., Wu, X., and Liang, T., "Three-dimensional identification of microorganisms using a digital holographic microscope,” Comput. Math. Methods Med. 2013, 162105 (2013).

[20] Javidi, B., Rawat, S., Komatsu, S., and Markman, A., "Cell identification using single beam lensless imaging with pseudo-random phase encoding,” Opt. Lett. 41(15), 3663 (2016).

[21] Javidi, B., Markman, A., Rawat, S., O’Connor, T., Anand, A., and Andemariam, B., "Sickle cell disease diagnosis based on spatiotemporal cell dynamics analysis using 3D printed shearing digital holographic microscopy,” Opt. Express 26, 13614 (2018).

[22] Mazarevica, G., Freivalds, T., and Jurka, A., "Properties of erythrocyte light refraction in diabetic patients,” J. Biomed. Optics 7, 244-247 (2002).

[23] Okamoto, F., Sone, H., Nonoyama, T., and Hommura, S., "Refractive changes in diabetic patients during intensive glycaemic control,” Br. J. Ophthalmol. 84, 1097-1102 (2000).

[24] Barer, R., "Interference Microscopy and Mass Determination,” Nature 169(4296), 366-367 (1952).

[25] Doblas, A., Sánchez-Ortiga, E., Martínez-Corral, M., and Garcia-Sucerquia, J., "Study of spatial lateral resolution in off-axis digital holographic microscopy,” Optics Communications 352, 63-69 (2015).

[26] Cuche, E., Marquet, P., and Depeursinge, C., "Spatial filtering for zero-order and twin-image elimination in digital off-axis holography,” Appl. Opt. 39, 4070-4075 (2000).

[27] Goodman, J.W., [Introduction to Fourier optics], W. H. Freeman (2017).

[28] Doblas, A., Sánchez-Ortiga, E., Martínez-Corral, M., Saavedra, G., Andrés, P., and Garcia-Sucerquia, J., "Shiftvariant digital holographic microscopy: inaccuracies in quantitative phase imaging,” Opt. Lett. 38(8), 1352-1354 (2013).

[29] Doblas, A., Sánchez-Ortiga, E., Martínez-Corral, M., Saavedra, G., and Garcia-Sucerquia, J., “Accurate singleshot quantitative phase imaging of biological specimens with telecentric digital holographic microscopy.,” J. Biomed. Optics 19(4), 046022 (2014).

[30] Sánchez-Ortiga, E., Doblas, A., Saavedra, G., Martl’inez-Corral, M., and Garcia-Sucerquia, J., “Off-axis digital holographic microscopy: practical design parameters for operating at diffraction limit,” Appl. Opt. 53(10), 20582066 (2014).

[31] Colomb, T., Kühn, J., Charriere, F., and Depeursinge, C., "Total aberrations compensation in digital holographic microscopy with a reference conjugated hologram,” Opt. Express 14, 4300-4306 (2006).

[32] Di, J., Zhao, J., Sun, W., Jiang, H., and Yan, X., "Phase aberration compensation of digital holographic microscopy based on least squares surface fitting,” Opt. Commun. 282, 3873-3877 (2009).

[33] Seo, K.W., Choi, Y.S., Seo, E.S., and Lee, S.J., "Aberration compensation for objective phase curvature in phase holographic microscopy,” Opt. Letters 37, 4976-4978 (2012).

[34] Hollander, M., and Wolfe, D.A., [Nonparametric statistical methods], Wiley (1999).

[35] Randolph, K.A., and Myers, L.L., [Basic statistics in multivariate analysis], Oxford University Press (2013).

[36] Sánchez-Ortiga, E., Doblas, A., Saavedra, G., Martínez-Corral, M., and Garcia-Sucerquia, J., "Microscope, method and computer program for obtaining quantitative study of transparent samples by digital holographic microscopy,” in US Pat. 2016025288 (2016).

[37] Gallagher, E.J., Le Roith, D., and Bloomgarden, Z., "Review of hemoglobin A1C in the management of diabetes,” J. Diabetes 1, 9-17 (2009). 